\title{
A Spiral Spring Electrostatic Actuator for Double Layer Deformable Mirror with Continuous Phase Sheet
}

\author{
Yu Zhou, Cyrus Shafai, and James Dietrich \\ Electrical and Computer Engineering, University of Manitoba, Winnipeg, M.B., Canada, R3T 5V6
}

\begin{abstract}
A new electrostatic actuator for a double layer deformable mirror with continuous phase sheet is presented. The electrostatic actuator employs an upper electrode consisting of multiple Archimedean spiral spring arms supporting a small mass central attachment point, and is capable of separate the parameters of actuation voltage, spring constant, and resonance. The feature reduces the complexity in the designing of a double layer deformable mirror with continuous phase sheet that are widely employed in the adaptive optics area of astronomy. The fabricated device has a diameter of $1250 \mu \mathrm{m}$ with 4 single crystal silicon spiral arms each having a length of $6030 \mu \mathrm{m}$, thickness of $15 \mu \mathrm{m}$, and width of $30 \mu \mathrm{m}$. The actuator was simulated and tested to have a resonant frequency above $2600 \mathrm{~Hz}$, and was simulated to be capable of reaching approximately $5 \mu \mathrm{m}$ displacement with a drive voltage of less than $30 \mathrm{~V}$ with an actuator-electrode separation of $15 \mu \mathrm{m}$, based on performance parameter found experimentally.
\end{abstract}

Keywords: Electrostatic actuator, Spiral-shape spring, pico-second UV laser, Laser milling.

Yu Zhou, Cyrus Shafai, James Dietrich, "Spiral spring electrostatic actuator for double-layer deformable mirror with continuous phase sheet,” J. Micro/Nanolith. MEMS MOEMS 18(2), 025501 (2019), doi: 10.1117/1.JMM.18.2.025501.

Copyright 2019 Society of Photo-Optical Instrumentation Engineers. One print or electronic copy may be made for personal use only. Systematic reproduction and distribution, duplication of any material in this paper for a fee or for commercial purposes, or modification of the content of the paper are prohibited.

\section{INTRODUCTION}

Electrostatic actuation is one the most implemented driving mechanisms for MEMS applications [1]. The working principle of an electrostatic actuator is well understood, they offer low power operation in steady state, and its dynamic response time can be very high depending on its structure. However, electrostatic actuators can suffer from the need for large driving voltages, particularly if they are located far from their actuation electrode.

In the adaptive optics area for astronomy there is often need for large stroke actuators. There are various mechanisms for actuation, with electrostatic actuation offering a benefit of near zero steady state holding power. However, electrostatic actuators require large voltage if high resonant frequency $(>1 \mathrm{kHz})$ is also needed. Electrostatic actuated deformable mirrors have been explored by many groups [2-10]. In [2], T. Wu et al. demonstrated an electrostatic actuator using a bimorph cantilever as restoring mechanism which offered $3.8 \mu \mathrm{m}$ displacement at $130 \mathrm{~V}$. Fangrong $\mathrm{Hu}$ et al. [3] developed a dual-directional electrostatic actuated micromirror with a size of approximately $500 \mu \mathrm{m} \times 500 \mu \mathrm{m}$ made out of polysilicon. The DM was reported to have a resonant frequency of $\sim 2.5 \mathrm{kHz}$, and a maximum $1.65 \mu \mathrm{m}$ displacement at $100 \mathrm{~V}$. Both of the DMs above are digital mirrors with discontinuous phase sheet. In the applications of astronomy, DMs with continuous phase sheet are generally preferable. Jen-Liang Wang et al. [4] introduced an electrostatic actuated DM with a continuous polymer membrane for optical focusing. The DM had a $25 \mu \mathrm{m}$ deflection at $160 \mathrm{~V}$, but its focusing time was $14 \mathrm{~ms}$. A large stroke micro DM was introduced by Po-Yu Lin et al. [5]. The DM also had a polymer phase sheet of $25 \mathrm{~mm} \times 25 \mathrm{~mm}$, and was tested to have a maximum displacement of $39 \mu \mathrm{m}$ at $195 \mathrm{~V}$. However, its resonant frequency was $40 \mathrm{~Hz}$. The micromachined DM of Sarah J. Lukes et al. [6] was fabricated from a $3 \mathrm{~mm} \times 4.24 \mathrm{~mm}$ elliptical SU-8 membrane with a thickness of $2.5 \mu \mathrm{m}$. It had a maximum deflection of $14.8 \mu \mathrm{m}$ at $320 \mathrm{~V}$. Wenying Ma et al. [7] built a surface micromachined micro DM based on hexagonal parallel plate electrostatic actuator. The actuator array had a pitch of $408 \mu \mathrm{m}$, and was measured to have a $0.68 \mu \mathrm{m}$ stroke at a drive voltage of $30 \mathrm{~V}$.

Compared to the single layer DM with continuous phase sheet, the double layer DM with continuous phase has an advantage of potentially controllable compliance between adjacent locations of the phase sheet. T. G. Bifano et al. [8] reported a high stiffness and high resonance double layer deformable mirror (DM) system driven by electrostatic actuators with a $0.8 \mu \mathrm{m}$ displacement 
requiring over $100 \mathrm{~V}$ driving voltage. The double layer structure uses a lower electrostatic actuator, whose movable membrane is attached to an upper layer deformable mirror. The design was fabricated by Boston Micromachines Corporation, and later, improved to give a $1.5 \mu \mathrm{m}$ displacement with a response time $<20 \mu$ s [9]. However, the driving voltage was reported to be in 100 's of volts. Il Woong Jung et al. [10] successfully fabricated a single-crystal-silicon continuous membrane deformable mirror array with $200 \mu \mathrm{m}$ pitch. The element of the array was capable of deflecting $125 \mathrm{~nm}$ at $100 \mathrm{~V}$, and had a resonant frequency at $25 \mathrm{kHz}$.

In case of double layer deformable mirror designs with continuous phase sheet, the moving membrane of the actuator is located at an intermediate position and is attached to the upper mirror phase sheet (see Figure 1). This is similar to the structure employed by Boston Micromachines Corp.. Figure 1 shows the cross-section of a DM with 3 elements in row and its phase sheet attached. Consider Element 2, which has its MEMS platform anchored at the spacer and spring anchor mounts, while the attached phase sheet mirror is effectively anchored at the bonding pillars of Element 1 and Element 3 (labeled A and C in the figure). This means that Elements 1,2, and 3 are connected mechanically.

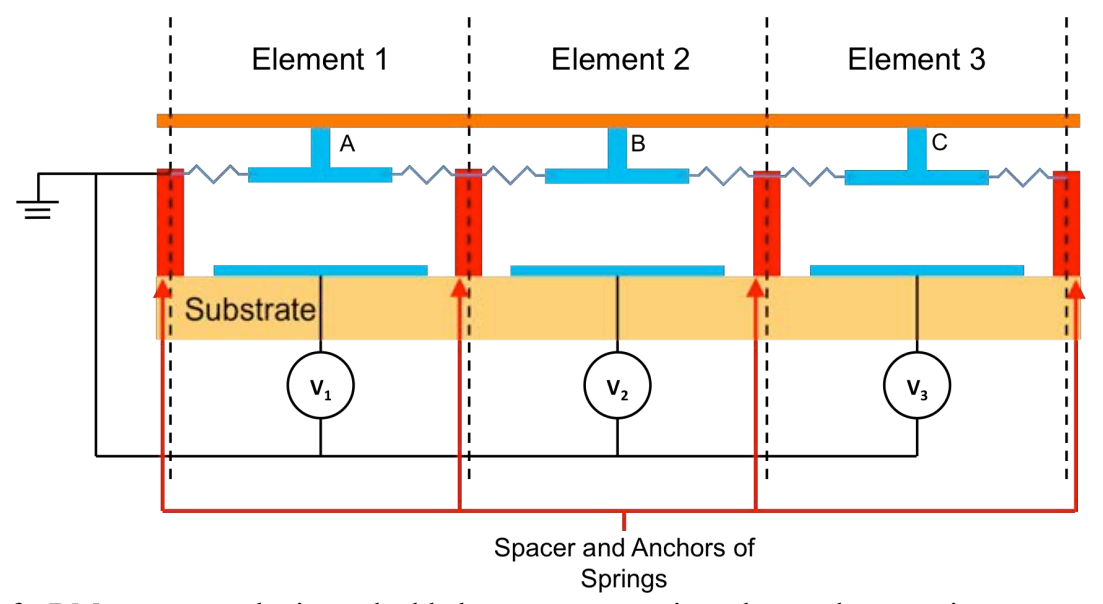

Figure 1. Schematic view of a DM system employing a double layer structure using a lower electrostatic actuator, whose movable membrane is attached to an upper layer deformable mirror. Each actuator membrane is anchored to the spacer (red), while the phase sheet mirror is attached to the actuator at points $\mathrm{A}, \mathrm{B}$, and $\mathrm{C}$.

Consider when Element 2 is activated while Element 1 and 3 are inactive. In this case, the spring constant of Element 2 should be calculated as a sum of the spring constant of the upper electrode and the phase sheet above it. However, we should also consider the case when both Element 1 and 3 are activated and Element 2 (which was originally activated) is then switched off. Noticing that an electrostatic actuator normally can only do pulling motion, therefore, the restoration force is solely generated by the suspending springs. As a result, the effective spring constant equals the spring constant of the upper electrode subtracted from the effective spring constant of the phase sheet.

The change in the total spring constant for the two above cases, leads to a varying resonant frequency in different types of motions, which is undesirable. A solution to this problem is to design the upper electrode with a much higher spring constant than the phase sheet, therefore the variation in the spring constant is reduced.

Therefore, employing a double layer deformable mirror design adds another layer of complexity to the system, since the designer should not only consider the spring constant of the actuator itself, but also the effective spring constant of the phase sheet. Together these two parameters can give favorable mechanical compliance between two adjacent locations on the mirror phase sheet. While difficult to achieve in practice, careful design can give controllable compliance between adjacent locations on the mirror phase sheet.

The application focus of the research presented in this paper is a large stroke and low voltage electrostatic actuator for a double layer deformable mirror system. A 2D array of 5-by-5 actuators was fabricated from a $1.2 \mathrm{~cm} \mathrm{x} 1.2 \mathrm{~cm}$ and $15 \mu \mathrm{m}$ thick silicon membrane using a laser micromachining. This particular application required relatively large displacement electrostatic actuators $(\sim 5 \mu \mathrm{m})$, with a resonant frequency $>2 \mathrm{kHz}$, and a low drive voltage $(<30 \mathrm{~V})$. None of the DMs mentioned above can meet all three requirements simultaneously. These performance requirements were achieved by replacing the membrane of the electrostatic actuator in the double layer deformable mirror design with spiral elements. Each element acts both as spring and upper electrode for the actuator, with the individual spiral springs connected at a small central attachment point of minimal mass (see Figure 2). This configuration results in the bottom electrode having most of its force applied to the spring arms, as opposed 
to the central mass.

This structure has the further benefit of greatly simplifying the design process, since it enables the separation of the design parameters of actuation voltage, spring constant, and resonance due to:

- The total system spring constant scales with the number of spiral arms.

- The total system mass scales with the number of spiral arms, when a small mass central attachment point is used.

- The facing area of the spring elements scales with the number of spiral arms, and so the electrostatic pulling force scales similarly.

- The spiral shape itself enables compact size of spring structure, interleaving of multiple springs (allowing for integer changes in spring constant), and rotation during deflection to minimize non-linear response over large deflection

Each individual spiral element can then be designed for a desired resonance frequency and stoke vs. force relationship, and the overall system spring constant of the upper spiral electrode can be simply changed by varying the number of spring arms. This provides freedom in changing the spring constant of the actuator, in order to get a desirable DM system compliance, without affecting the drive voltage and resonant frequency of the DM system.

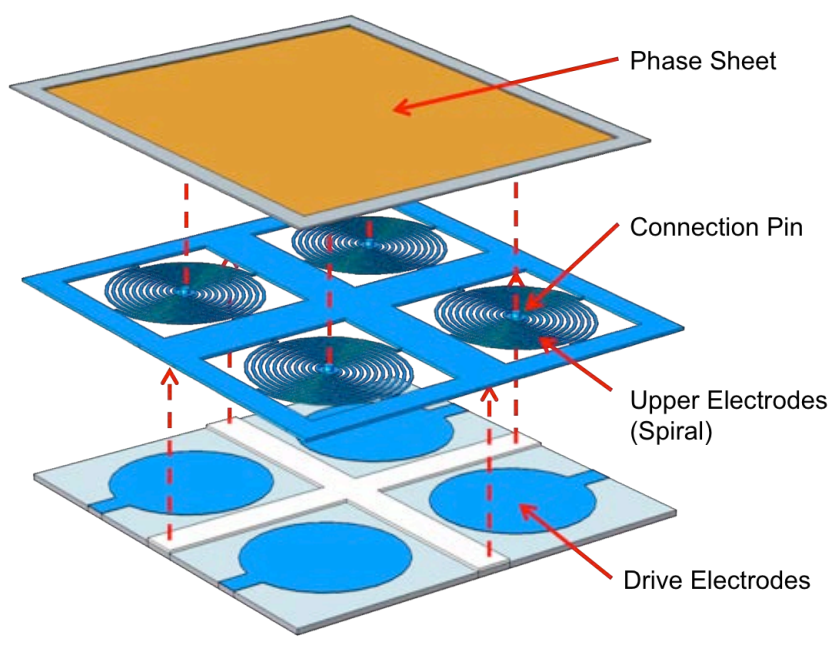

Figure 2. Schematic of AO system with spiral actuators.

\section{DESIGN AND SIMULATIONS}

A common design for an electrostatic actuator is a parallel plate system, which has the parameters of actuation voltage, spring constant, and resonance all interrelated. This complicates the design of the MEMS structure, since changing one parameter affects the others. Consider a parallel-plate electrostatic actuator shown in Figure 3, the membrane is supported by springs of spring constant $k$. The membrane of mass $m$ is dominant compared to the springs. The underlying driving electrode and membrane, both of surface area $A$, are separated by a distance $d$, from which the actuation force is set by the drive voltage. The deflection $\Delta d$ of the actuator is given by Equation 1, with its resonant frequency $f$ given in Equation 2.

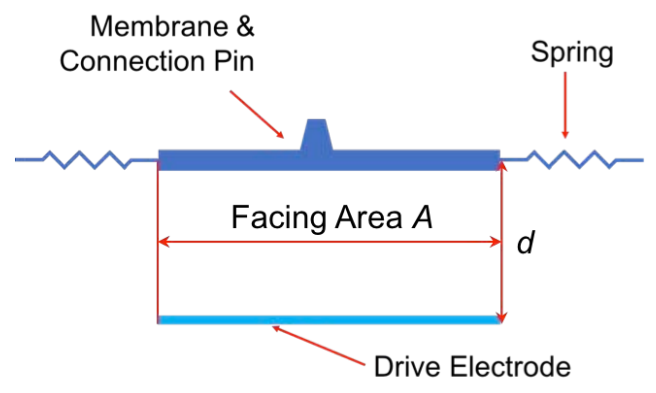

Figure 3. Conventional electrostatic actuator set-up. 


$$
\begin{gathered}
\Delta d=\frac{F_{e}}{k}=\frac{1}{2} \varepsilon \frac{A V^{2}}{k(d-\Delta d)^{2}} \\
f \propto \sqrt{\frac{k}{m}}
\end{gathered}
$$

where $F_{e}$ is the electrostatic force. Conventionally, the restoration force of the actuator is decided by the physical property of the springs, so does the resonant frequency $f$. For a large deflection low voltage electrostatic actuator, a softer spring system is required. However, according to Equation 2, the resonance frequency is proportional to the square root of the spring constant over mass. As a result, a softer spring with the same mass, which is mainly decided by the center membrane, leads to a lower resonance frequency, which is undesirable.

As shown in the discussion above, a commonly designed electrostatic actuator is not optimal for the applications of double layer DMs with continuous phase sheet, as all three major design parameters are all interrelated. To separate these three design parameters, we present a spiral shaped electrostatic actuator with its spiral shape of the springs calculated based on an Archimedean spiral following Equation 3:

$$
R=R_{0}+R_{i} \cdot \theta
$$

where $R$ is the total radius, $R_{0}$ is the initial radius, $R_{i}$ is the radius increment per degree of arc, and $\theta$ is the degree of arc.

The Archimedean spiral shape has been investigated and implemented by multiple research groups [11-16]. Both Narito Shibaike [11] and William C. Tang et al. [12] used the spiral spring as the restoring mechanism in the micro-rotating actuators. Yen-Ru Huang et al. [13] reported a carbon-nanotube-based parallel capacitor implementing the Archimedean spiral shape to increase the facing area of the capacitor. Jinghong Chen et al. [14] designed a micro-inductor with a spiral shape, while Tetsuo Kan et al. [15] presented a MEMS polarizer with spiral shape actuated by nitrogen gas pressure. None of above is an actuator with motion in out-of-plane direction. Chenye Yang et al. [16] presented an out-of-plane PZT bimorph actuator with Archimedean spiral actuator tethers. The actuator was reported to have a $17 \mu \mathrm{m}$ displacement with a $110 \mathrm{~V}$ drive voltage. However, the actuator has a diameter of $19 \mathrm{~mm}$, which limits its application in applications with confined actuator size such as deformable mirror array. Fukushige et al. [17] reported a conical inductor made of Pd-based TFMG $\left(\mathrm{Pd}_{76} \mathrm{Cu}_{7} \mathrm{Si}_{17}\right)$ that is capable of a tuning range of 3.64 to $3.75 \mathrm{nH}$ at $2 \mathrm{GHz}$, although it is only used as a two state actuator. An electrostatic actuator based on the same structure was also reported by this group [18]. The actuator has a diameter of $600 \mu \mathrm{m}$, and a stroke of $180 \mu \mathrm{m}$. Again, the stroke is not controllable as pull-in occurs when the actuator is activated. Hu et al. [19] presented a conical electrostatic actuator made of polysilicon. The actuator has a side length of more than $250 \mu \mathrm{m}$, and was reported to have $2.7 \mu \mathrm{m}$ at $50 \mathrm{~V}$, while the resonant frequency is not reported. None of the above works has investigated the unique advantages of the spiral shape spring for electrostatic actuation for an out-of-plane electrostatic actuator.

Figure 4 shows a schematic cross-section of the of the designed spiral upper electrode with a diameter $2 R=1250 \mu \mathrm{m}$. Each spiral arm was designed with an initial radius $R_{0}=80 \mu \mathrm{m}$, and a radius increment per degree of $\operatorname{arc} R_{i}=210 \mu \mathrm{m}$, giving a final length of $6030 \mu \mathrm{m}$.

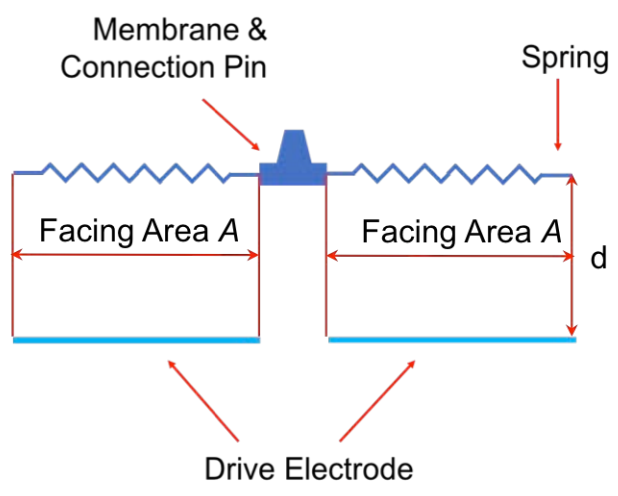

Figure 4. The cross-section of the schematic of the spiral actuator.

Equations 4 and 5 are the relevant equations for the spiral actuators and are based on Equations 1 and 2, but with $N$ spring 
elements in each. In Equation 5, $k$ stands for the spring constant of each individual spring, $m$ is the mass of each individual spring, and $M$ is the mass of the center connection location. If designed such that $M$ is much smaller than $N m$, the central spring has minimum effect on the resonance frequency. The total spring constant of the upper electrode $k_{\text {total }}$ is given in Equation 6 . We can see that in either case (neglecting any fringing electric field), the presence of the $N$ springs has no net effect on displacement and drive voltage, and resonance. This is because the surface area $(A)$ of the combined spirals increases in accordance with the number of spiral elements $(N)$.

$$
\begin{gathered}
\Delta d=\frac{F_{e}}{N k}=\frac{1}{2} \varepsilon \frac{N A V^{2}}{N k(d-D)^{2}}=\frac{1}{2} \varepsilon \frac{A V^{2}}{k(d-\Delta d)^{2}} \\
f \propto \sqrt{\frac{N k}{N m+M}} \approx \sqrt{\frac{k}{m}} \text { if } M \ll N m \\
k_{\text {total }}=N k
\end{gathered}
$$

Figure 5 shows three related spiral designs, with their simulated performance. Simulations were done using COMSOL Multiphysics software [20]. In Figure 5, $f$ indicates the resonant frequency of each structures, and $k_{\text {total }}$ is the spring constant of each structures. From Figure 5 we can see that despite the dramatic change in the spring constant, from $3.28 \mathrm{~N} / \mathrm{m}$ to $6.64 \mathrm{~N} / \mathrm{m}$ caused by varying the number of spiral beams, the deflection and resonant frequency of all three devices are very similar. It should be noted that the slight deviation in the resonant frequency and the deflection is largely due to the existence of the center connection stage of the spiral arms, as the size of this element is the same in all three designs. For the 4 arm design of Figure 5(c), the next four higher order resonant frequencies found by simulation are at $5024.6 \mathrm{~Hz}, 6003.3 \mathrm{~Hz}, 6599.7 \mathrm{~Hz}$, and 6601.4 $\mathrm{Hz}$.

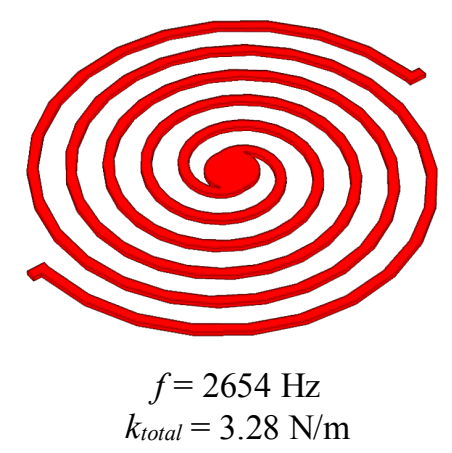

(a)

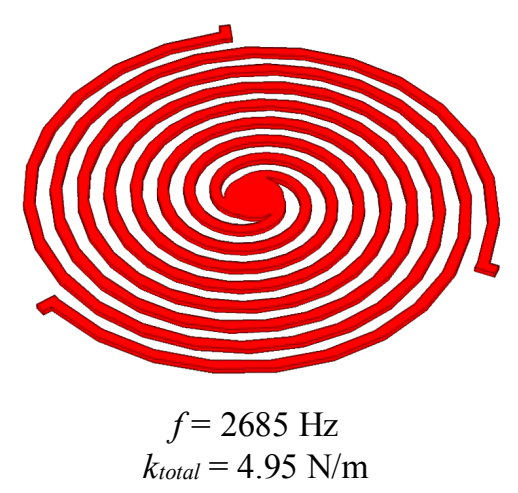

(b)

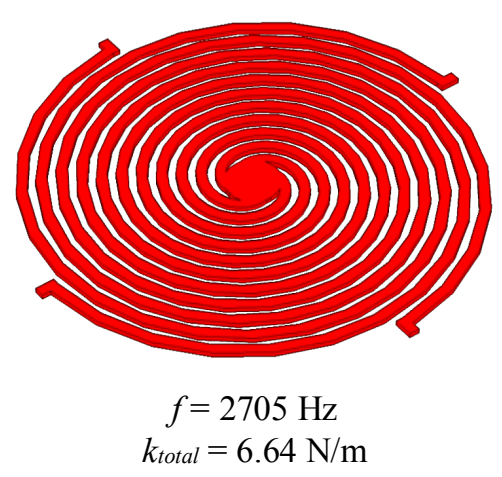

(c)

Figure 5. Comparison of resonant frequency $(f)$ and spring constant $\left(k_{\text {total }}\right)$ between spiral upper electrodes with different number of arms $((a)$ has 2 spriral arms, (b) has 3 spriral arms, (c) has 4 spriral arms). All have silicon spiral springs that are $30 \mu \mathrm{m}$ wide, $15 \mu \mathrm{m}$ thick, $6030 \mu \mathrm{m}$ long, an $80 \mu \mathrm{m}$ wide central platform, and the diameter of the structure is $1250 \mu \mathrm{m}$.

These actuators of this paper were designed for use with the polymer DM phase sheet mirror that was descibed in [21]. The phase sheet was a $12 \mathrm{~mm} \times 12 \mathrm{~mm} \mathrm{Al} / \mathrm{SU}-8 / \mathrm{Al}$ membrane mirror. The mirror is constructed from a $4 \mu \mathrm{m}$ thick SU-8 film, that was coated on both sides by $250 \mathrm{~nm}$ thick thermally evaporated aluminum. In [21], an experimental verification was done to verify the shase sheet's spring constant. The $12 \mathrm{~mm} \times 12 \mathrm{~mm}$ phase sheet was anchored on its sides, and an underlying MEMS a Lorentz force actuator was used to force a deformation at its centre. The combined spring constant (DM phase sheet and actuator together) was measured to be ranging from $0.0718 \mathrm{~N} / \mathrm{m}$ to $0.116 \mathrm{~N} / \mathrm{m}$. The spring constant of the Lorentz force actuator itself was measured to be approximately $0.1 \mathrm{~N} / \mathrm{m}$. We can see that the spring constant of the $12 \mathrm{~mm} \times 12 \mathrm{~mm}$ phase sheet is on the order of $0.01 \mathrm{~N} / \mathrm{m}$, which is considerably smaller than the spring constant of actuators of Figure 5 and so meeting the required criteria stated in section 1 .

The phase sheet was also investigated in this paper, in simulation, to explore the spring constant on the phase sheet under two conditions. The first explored the stiffness of the entire phase sheet unloaded by actuators. This simulation had the phase sheet anchored on all sides, and actuated by a single force point pushing at its center. The simulation gave a $0.0150 \mathrm{~N} / \mathrm{m}$ spring constant for the entire phase sheet. The second simulation explores the effective spring constant of an element of the phase sheet above a single actuator, loaded by adjacent actuators. This represents the mechanical compliance condition that would be present in an actuator array, which would effectively sub-divide the phase sheet, and so stiffen it. The set-up of the simulation is shown 
in Figure 6, with the central point (Actuator 1) surrounded by 8 other actuators. This simulation was done to explore the situation where surrounding actuators are at rest, and so act to stiffen the phase sheet locally. In this simulation, the neighboring actuators are set as rigid boundary points fixing the phase sheet. In reality, however, these adjacent actuators would have some compliance. This simulation found that the phase sheet at the Actuator 1 location had an effective spring constant at $0.135 \mathrm{~N} / \mathrm{m}$. This is again much less than the spring constant of the spiral actuators, and so meeting the required criteria stated in section 1.

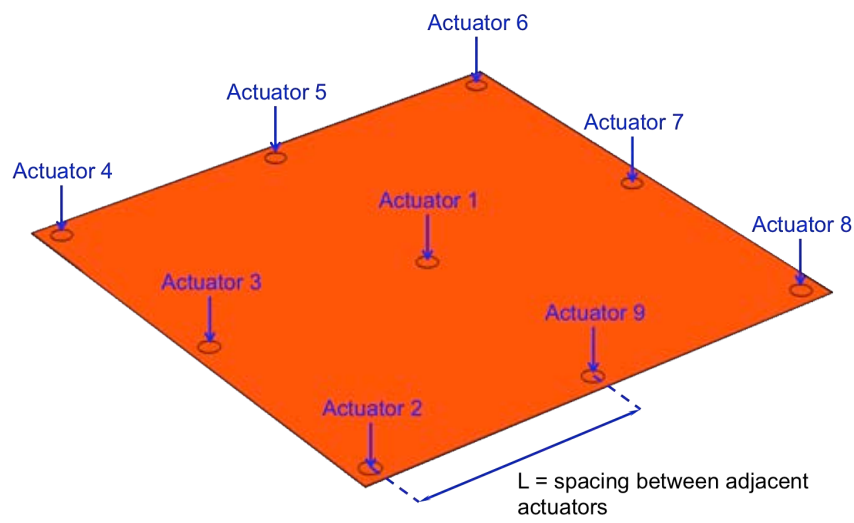

Figure 6. Structure used in the simulation of the phase sheet effective spring constant above a single actuator. The center actuator (Actuator 1) was set as active while the surrounding actuators were set as fixed. $L$ stands for the spacing between two adjacent actuators and is $1800 \mu \mathrm{m}$ according to the actuator design.

From equations 1 and 4, we can see that for an electrostatic actuator the electrostatic force generated is proportional to the inverse of the square of the distance $d$ separating the moving and fixed electrodes. An electrostatic actuator also has a pull-in distance, which is the maximum controllable stroke of the actuator. For a typical electrostatic actuator shown in Figure 3, the pull-in occurs at approximately $1 / 3$ of the original separation [22]. As a result, to maximize the displacement of the actuator with a limited drive voltage, simulation was done with a separation between the upper (spiral) electrode and bottom (drive) electrode $d=15 \mu \mathrm{m}$, which is 3 times of the required stroke $(5 \mu \mathrm{m})$.

Figure 7 shows the simulated displacement versus drive voltage performance of the specific actuators with 2, 3, and 4 spiral arms shown in Figure 5. We can see that these structures perform similarly in displacement vs. applied voltage, even though each has different spring constant. All are capable of achieving $\sim 5 \mu \mathrm{m}$ displacement with a drive voltage of under $30 \mathrm{~V}$, which is the specific requirement of this project. Beyond $30 \mathrm{~V}$ the actuator was found to pull-in. We can, however, see a slight increase in voltage needed for cases with more spring arms. This is because less electric field energy couples to each individual spiral arm, and this is due to adjacent spirals limiting the fringing energy on to the sides of an individual actuator arm.

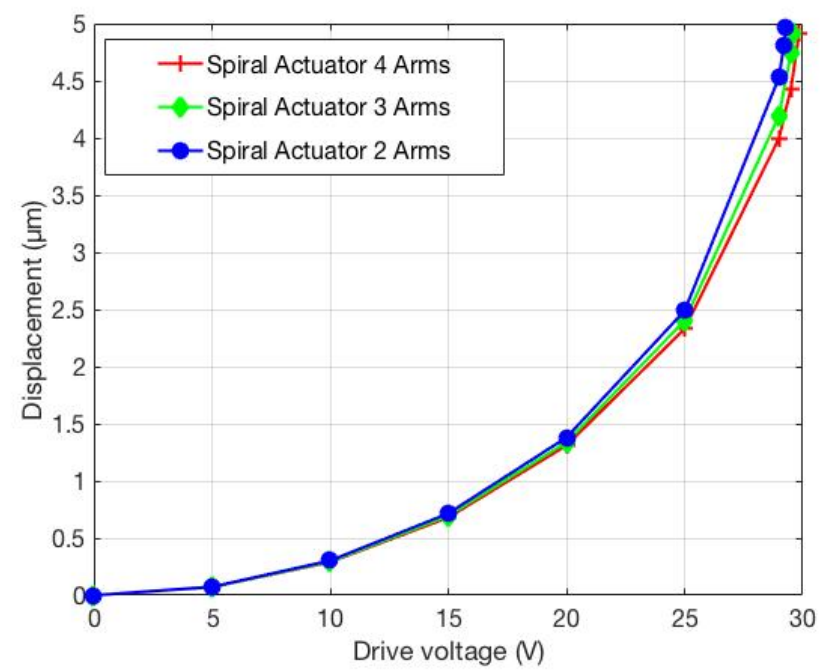

Figure 7. Simulated displacement of the spiral actuator with spiral arms of $30 \mu \mathrm{m}$ wide, $15 \mu \mathrm{m}$ thick, $6030 \mu \mathrm{m}$ long, and an $80 \mu \mathrm{m}$ wide central platform versus the drive voltage. The simulation shows that this structure is capable of achieving $\sim 5 \mu$ displacement with a drive voltage of under $30 \mathrm{~V}$ for the actuators with 2,3 , and 4 arms. 


\section{FABRICATION}

The fabrication process of the electrostatic actuator array (the upper spiral electrode and drive electrode layers of Figure 2) is described in Figure 8. A two-wafer process is used, with $250 \mu \mathrm{m}$ thick (100) silicon wafers.

The spiral electrode wafer fabrication is shown in Figure 8. A $1 \mu \mathrm{m}$ thick layer of silicon dioxide (orange) is grown on the wafer (blue) using a wet thermal growth process at $1100^{\circ} \mathrm{C}$. Then the backside silicon dioxide is patterned and etched using wet etching. The $\mathrm{SiO}_{2}$ serves as the mask for the subsequent $\mathrm{KOH}$ etch. The wafer is then etched in $30 \% \mathrm{KOH}$ heated to $80{ }^{\circ} \mathrm{C} 200$ $\mu \mathrm{m}$ deep, leaving $50 \mu \mathrm{m}$ of silicon in the actuator locations (Figure 8(a)). Another thermal oxidation is then carried out to grow a $1 \mu \mathrm{m}$ thick layer of silicon dioxide (red) in the etching trench as the mask for the next etch (Figure 8(b)). The frontside silicon dioxide is then patterned (Figure 8(c)), and then etched in $\mathrm{KOH}$ in three successive steps to define the pillar, and to thin the membrane to $30 \mu \mathrm{m}$ at the locations of the support frames and $15 \pm 1 \mu \mathrm{m}$ at the locations of the spiral actuators (Figures 8(d), $8(\mathrm{e}), 8(\mathrm{f})$ ). This is followed by the removal of the silicon dioxide a deposition of $180 \pm 20 \mathrm{~nm}$ aluminum coating material (green) by DC sputtering (Figure $8(\mathrm{~g})$ ). The aluminum is patterned into actuator shape using wet etching. Following this, the spiral actuators are released (Figure 8(h)). Details of the actuator release are given in the section 3.1.

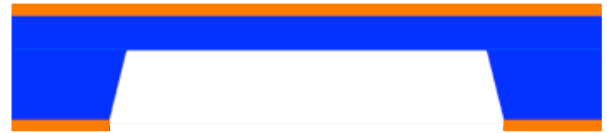

(a)

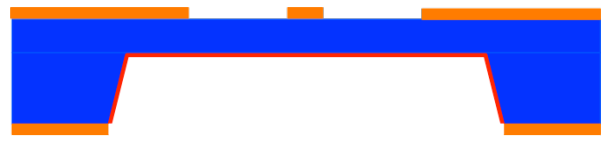

(c)

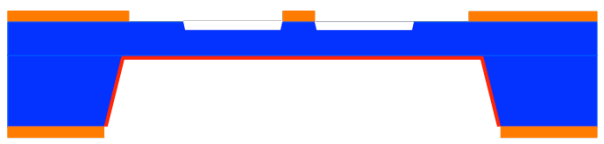

(e)

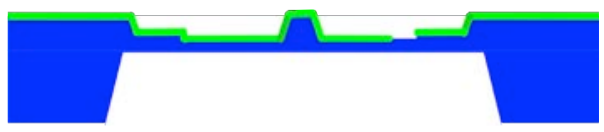

(g)

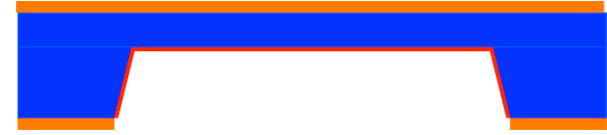

(b)

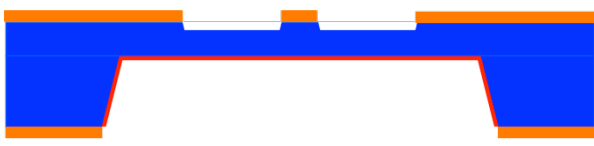

(d)

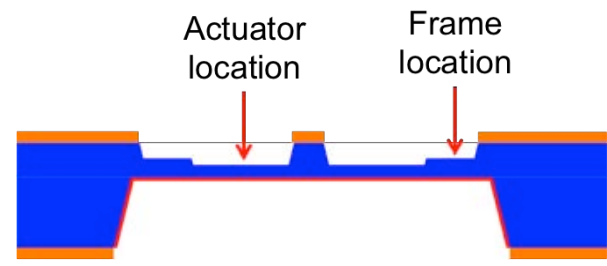

(f)

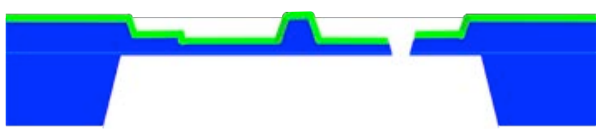

(h)

Figure 8. Fabrication process of the spiral electrode.

The fabrication of the drive electrodes on the second wafer is shown in Figure 9. Alignment trenches $185 \mu \mathrm{m}$ deep are etched into the frontside of the wafer to match the structure of the actuator wafer with the spirals, so to ensure they align during fabrication (Figure 9(a)). Etching is done using $\mathrm{KOH}$ with a $1 \mu \mathrm{m}$ thick thermally grown silicon dioxide mask. A second $\mathrm{KOH}$ etch is used to pattern a trench $15 \mu \mathrm{m}$ deep, which will later be the spacing between the spiral actuators and the drive electrodes (Figure 9(b)). This etch also deeps the first alignment trench to $200 \mu \mathrm{m}$ deep. The substrate is then thermally oxidized $(1 \mu \mathrm{m}$ thick) to provide electrical insulation, and $180 \pm 20 \mathrm{~nm}$ aluminum is DC sputtered on the frontside and is patterned into the structure of the drive electrodes (Figure 9(c)). Then a $1 \mu \mathrm{m}$ layer of $\mathrm{SiO}_{2}$ is sputtered as electrical insulation, using RF reactive sputtering from a silicon wafer target material. After fabrication, this wafer is attached to the underside of the spiral electrode wafer (Figure 9(e)). Figure 10 shows a picture of a fabricated bottom drive electrode wafer. 


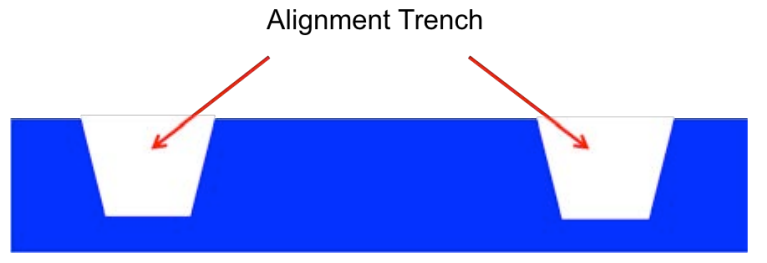

(a)

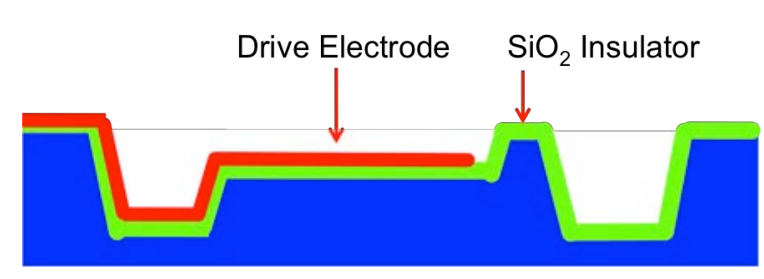

(c)

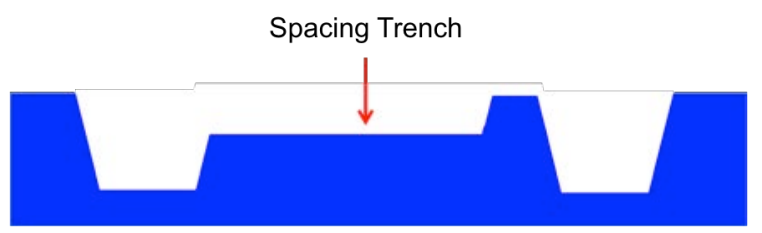

(b)

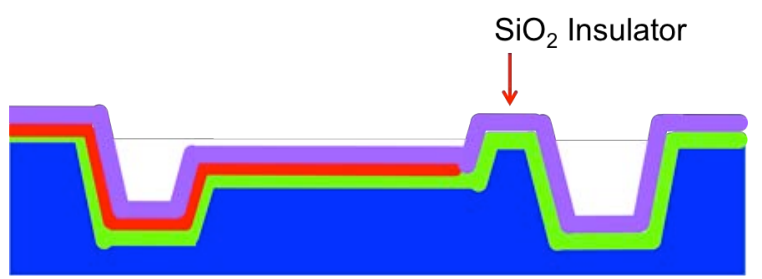

(c)

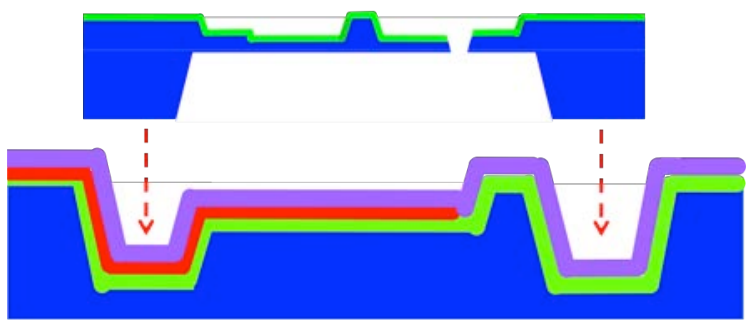

(e)

Figure 9. Fabrication process of the drive electrode.

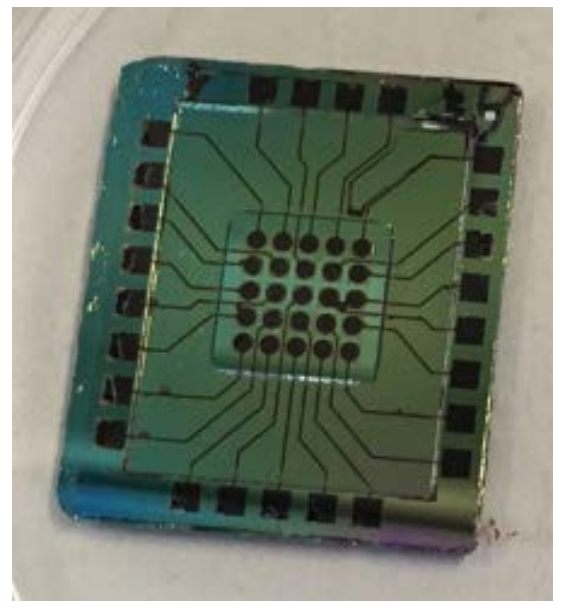

Figure 10. Fabricated bottom drive electrode of the actuator.

\subsection{Releasing of the Spiral Electrodes}

Two processes were investigated for releasing the spiral actuators. The first was a plasma etch process. This process used the aluminum metallization on the spiral arms as the etch mask. However, the plasma etch releasing process was not able to give a uniform etch profile over the surface of the device. This resulted in varying shape of the spiral features. Figure 11 illustrate this issue. The first problem was due to a heating issue in the etch. Because the structure is very thin compared to its diameter $(\sim 15$ $\mu \mathrm{m}$ in thickness compared to $1300 \mu \mathrm{m}$ in side-length for each spiral actuator and $9000 \mu \mathrm{m}$ for the whole $5 \times 5$ array), during etching the center becomes hotter than the edges (where the spiral meets the bulk silicon wafer). The etching process is then slower at the edges of the structure. The second problem occurs at the center of the spiral. The smaller mask opening at the 
center of the structure impeded the plasma from reaching the silicon surface, therefore, the etching rate is also slower in this location. Figure 11 shows a partially etched spiral structure under a microscope. In Figure 11, the silvery colored part is the spiral structure coated with aluminum, while the grey and black area is the partially etched silicon.

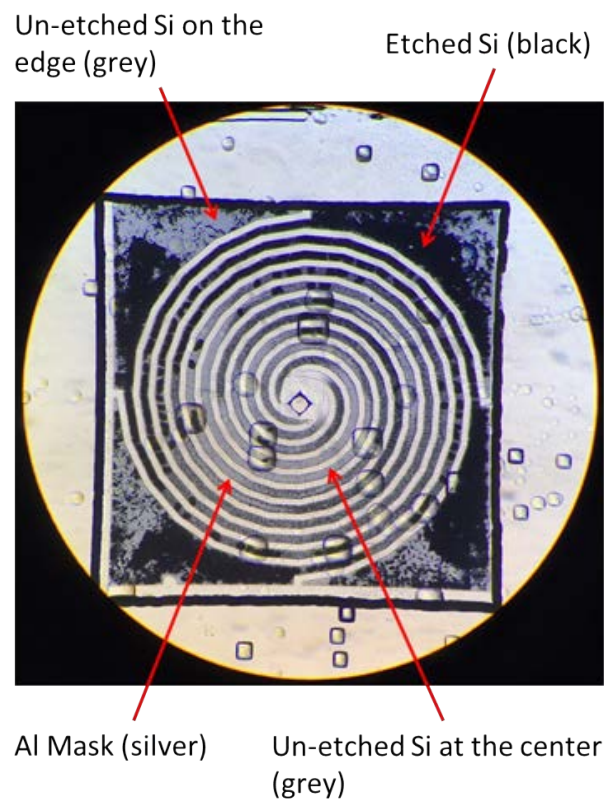

Figure 11. Surface of a partially etched spiral structure. The non-uniformity of the color (black and grey) shows the imperfections of the plasma etch.

\subsubsection{Laser Milling of the Spiral Electrodes}

A laser etching method was developed to pattern the actuators to avoid the above issues with the plasma etching process. An ultraviolet (355-nm) diode-pumped solid-state pico-second laser (A-355ps Laser Micromachining system) from Oxford Lasers Ltd. was used to release the structure.

The laser micromachining system employed a diode-pumped solid-state laser, which has a pulse energy peaking at $0.12 \mathrm{~mJ}$, a pulse duration of $6 \mathrm{ps}$, and a pulse frequency range from $1 \mathrm{~Hz}$ to $400 \mathrm{~Hz}$. The kerf width needed to be understood before laser cutting was done to free the actuators. Kerf width was tested over various attenuation sets. Table 3 shows the results of the test after 20 runs of milling process over the same cut area on a silicon wafer. A circular cut was made, $1 \mathrm{~mm}$ in diameter. This was done to observe possible non-circular nature of the laser spot. A frequency of $400 \mathrm{~Hz}$ was used, and a laser spot traveling speed was $1 \mathrm{~mm} / \mathrm{sec}$. We can see from the first entry of Table 1, that along the sides of the circle tangent to the $\mathrm{x}$ and $\mathrm{y}$ axes, the spot size is not uniform. As the power level was reduced from its peak of $0.12 \mathrm{~mJ}$ by changing the attenuation, we can see that the laser spot became more smaller and more uniform in relation to the cut size on the $\mathrm{x}$ and $\mathrm{y}$ axial direction. The most uniform kerf width was $18 \mu \mathrm{m}$ in $\mathrm{x}$-axis and $20 \mu \mathrm{m}$ in y-axis at $99.4 \%$ of attenuation. Further increasing the attenuation did not shrink the kerf width significantly nor did it improve the uniformity in the spot diameter. This might be a result of the non-linear control of the attenuator beyond $90 \%$, based on this laser's specification showing the attenuator only accurate from $0 \%$ to $90 \%$ of the energy attenuation [23]. Accordingly, this power level was used in the actuator fabrication, as it gave the best cutting performance in terms of achieving the best kerf. 
Table 3. Kerf width vs. Attenuation

\begin{tabular}{|c|c|c|}
\hline Attenuation & $\begin{array}{c}\text { kerf width tangent to } \\
\text { x-axis }(\mu \mathrm{m})\end{array}$ & $\begin{array}{c}\text { kerf width tangent } \\
\text { to y-axis }(\mu \mathrm{m})\end{array}$ \\
\hline $0 \%$ & 50 & 100 \\
\hline $90 \%$ & 30 & 40 \\
\hline $99 \%$ & 25 & 32.5 \\
\hline $99.4 \%$ & 18 & 20 \\
\hline
\end{tabular}

Laser cutting of the actuators first required the substrate to be clamped to an aluminum stage. The movement of the stage has a $0.1 \mu \mathrm{m}$ resolution and $\pm 1.5 \mu \mathrm{m}$ accuracy in both $\mathrm{x}$ and $\mathrm{y}$ axis. Figure 12 shows the inside set-up of the laser milling system. Full release of spiral features of the actuators required 25 runs of milling and a picture of the released upper electrode array is shown in Figure 13. In all runs, 99.4\% attenuation, $400 \mathrm{~Hz}$ pulse frequency, and $1 \mathrm{~mm} / \mathrm{s}$ moving speed was used.

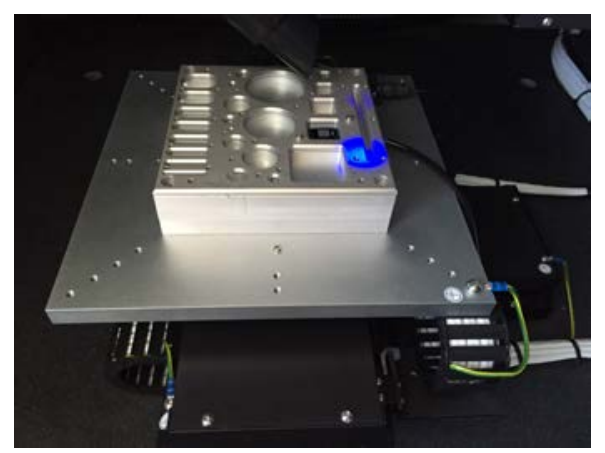

Figure 12. The aluminum jig and the stage.

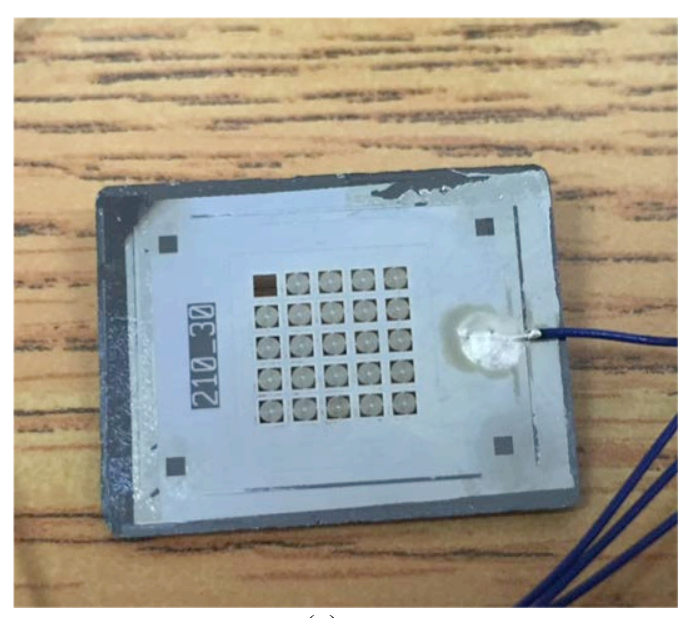

(a)

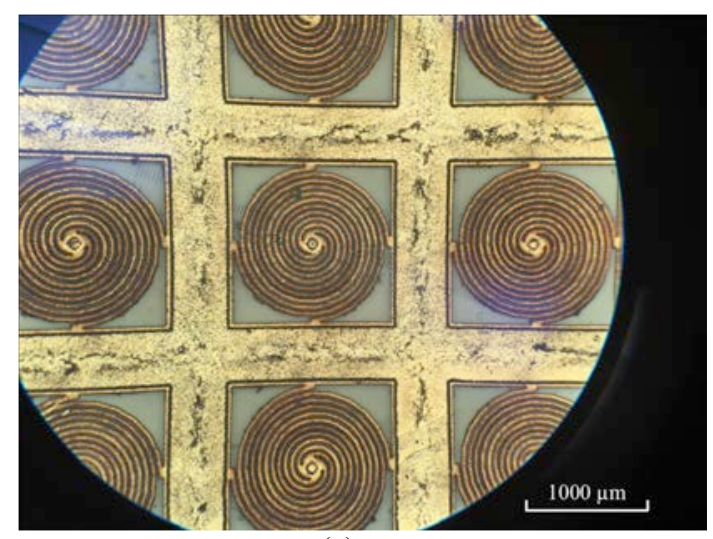

(c)

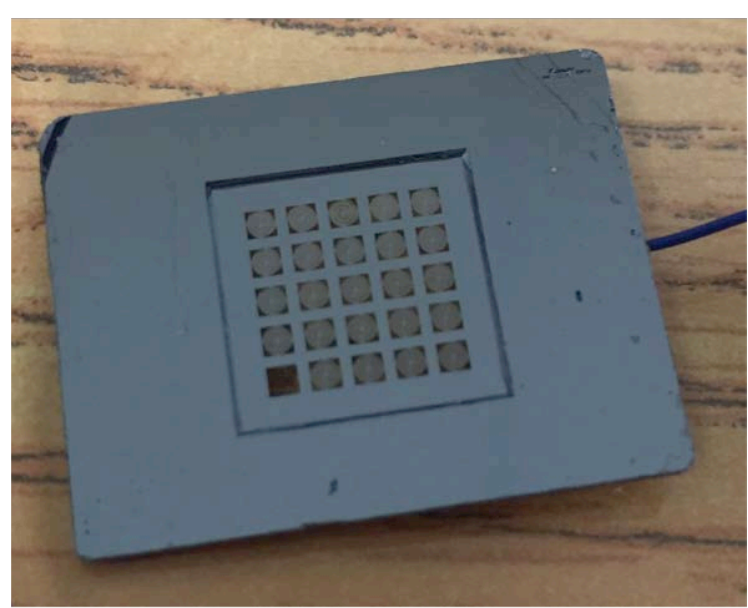

(b)

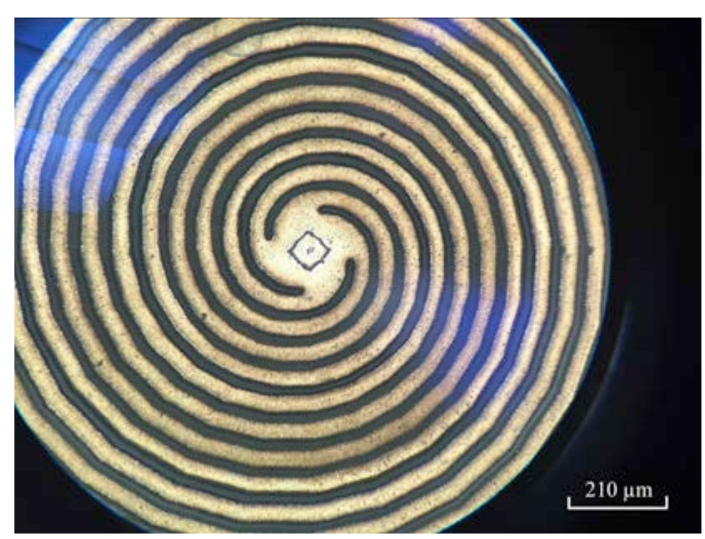

(d) 
Figure 13. (a) Front (phase sheet side) view of a released spiral upper electrodes array sample. (b) Back-side (side towards drive electrodes) view of the released array sample. (c) Picture of spiral upper electrode array under a microscope. (d) Single spiral upper electrode under $100 \times$ magnification.

\section{RESULTS AND DISCUSSION}

The actuator substrate was attached to the electrode substrate, and the separation between the actuator and drive electrode was measured by microscope. It was found that the two substrates were separated by $45 \pm 5 \mu \mathrm{m}$, and not the designed $15 \mu \mathrm{m}$. The possible reason for this might be that the laser milling process and assembly of the actuator were done outside of our cleanroom. As such, particle contamination could easily be present on the surfaces of the spiral upper electrode substrate and drive electrode substrate, so when attaching these substrates together their spacing was higher than expected.

The experimental set-up of Figure 14 was used for the actuator deflection tests. The MEMS structures require electrostatic actuation, however, higher dc voltages (over $60 \mathrm{~V}$ ) were found to result in charging of the dielectric upon the electrode pads. In order to avoid this, the device was testing using an ac source. A high frequency $(10 \mathrm{kHz})$ ac signal was used to provide an RMS equivalent dc voltage. The selection of $10 \mathrm{kHz}$ was chosen so as to be higher than the various resonances of the structure. The displacement of the actuator was observed and measured using microscope, by focusing the microscope on the upper surface of the center stage of the actuator before and after applying the drive voltage. By comparing the focal point of the microscope under both conditions, the static displacement of the actuator can be measured.

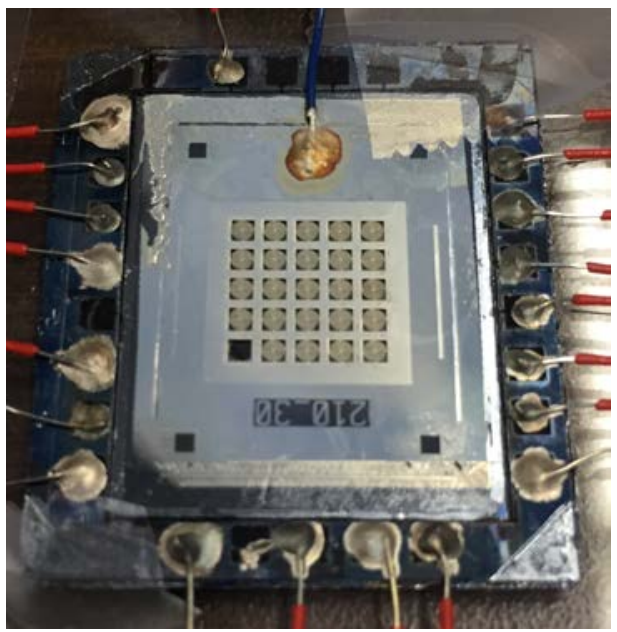

(a)

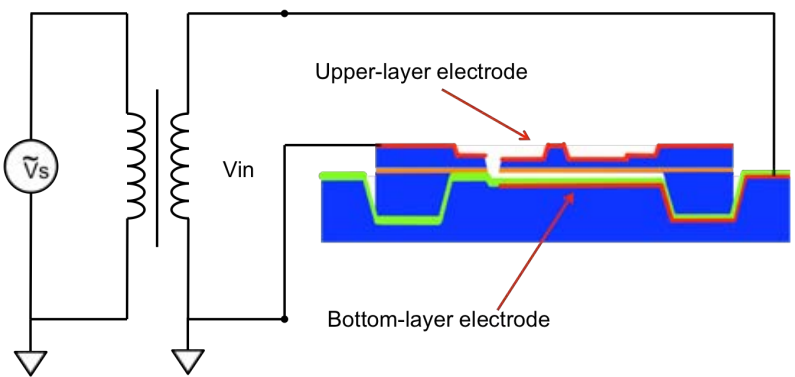

(b)

Figure 14. Experimental set-up of the testing sample.

The fabricated actuators were 4 spiral arm designs of Figure 5(c), which were shown in Figure 13. Figure 15 shows the performance of a tested actuator, and a comparison with simulated performance evaluated in COMSOL. For the simulation curve, new simulations were run for actuator to drive electrode spacings $d$ adjusted to be $45 \mu \mathrm{m}$, to match the measured electrode separation of the tested device. In Figure 15, the error bar of the measured data is set to be $\pm 1 \mu \mathrm{m}$ based on the measurement error of the microscope, while the error bar of the simulation result is based on simulations for device thicknesses of $15 \pm 1 \mu \mathrm{m}$ to represent error in fabrication of the silicon spiral arm thickness. 


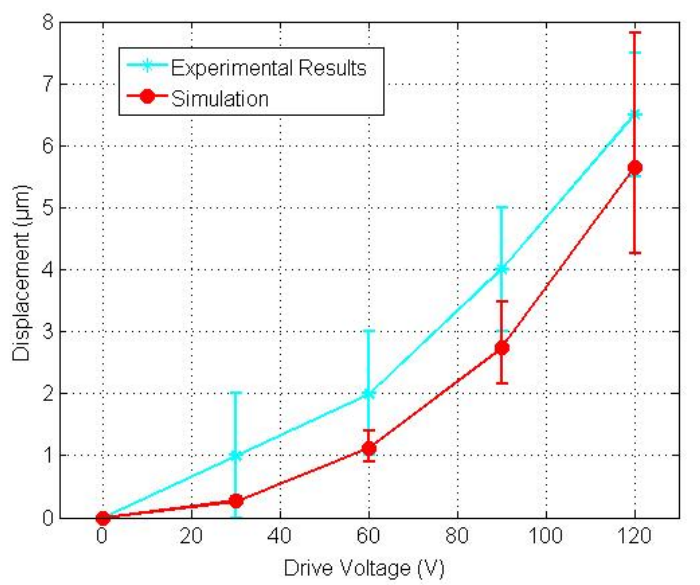

Figure 15. Comparison between experimental result and the simulation results for the fabricated spiral actuator. The red curve is the simulated result, and the blue curve is the measured result.

By applying the results in Figure 15 to Equation 1, the ratio of $k / A$ for the fabricated actuator can be calculated and compared to the $k / A$ of the simulated actuator of Figure 7. This is shown in Table 2. In Table 2, the last data point of Figure 15 is used to calculate $k / A$, since the error to measurement ratio is the smallest compared to other data points due to the larger deflection. The values in the bracket for the simulation column were calculated based on the thickness of $15 \pm 1 \mu \mathrm{m}$, while the values in the bracket in the measurement column were calculated based on the error bar of the measurement data. We can see close agreement between the simulated and measured of $k / A$ value for the actuator. This result validates the COMSOL model used to design the spiral actuator. We can then conclude that the simulation of Figure 7 is also accurate, which showed that the spiral actuator design is capable of achieving $\sim 5 \mu \mathrm{m}$ displacement with under $30 \mathrm{~V}$ drive voltage, if the separation $d$ were to be correctly fabricated at $15 \mu \mathrm{m}$.

Table 2. $k / A$ (ratio of spring constant vs. effective facing area) of the simulation results and measurements

\begin{tabular}{|c|c|}
\hline Simulation (Figure 7) & Measurement (Figure 15(b)) \\
\hline $6.577 \times 10^{6} \mathrm{~N} / \mathrm{m}^{3}$ & $6.6 \times 10^{6} \mathrm{~N} / \mathrm{m}^{3}$ \\
$\left(5.347 \times 10^{6} \mathrm{~N} / \mathrm{m}^{3}, 7.982 \times 10^{6} \mathrm{~N} / \mathrm{m}^{3}\right)$ & $\left(6.0 \times 10^{6} \mathrm{~N} / \mathrm{m}^{3}, 7.4 \times 10^{6} \mathrm{~N} / \mathrm{m}^{3}\right)$ \\
\hline
\end{tabular}

It should also be mentioned that the area of the bottom surface of this spiral actuator (which has 4 arms) is $7.39 \times 10^{-7} \mathrm{~m}^{2}$. If we assume the spring constant of the actuator is $k_{\text {total }}=6.64 \mathrm{~N} / \mathrm{m}$ as given in Figure 5, the effective surface area using the $k / A$ ratio in Table 2 is calculated to be $1.0 \times 10^{-6} \mathrm{~m}^{2}$. This is $37 \%$ larger than the bottom surface area of the spiral actuator. This is expected, as the electrostatic force would also couple to the sidewalls of the spiral structure. This is factor partially reduces the loss in surface area of the spiral structure vs. a solid electrode. The spiral itself has approximately a $60 \%$ fill factor, and so taking into account the sidewall coupling, it effectively has an approximately $85 \%$ fill factor from the point of view of electrostatic force coupling.

The first resonant frequency of the device was also measured to verify the performance of the actuator. Resonance movement study was done using a laser position measurement system to monitor spiral movement while it was driven with frequency swept ac source (shown in Figure 16). The actuation circuit was the same as the one shown in Figure 14. A Tektronix TDS 2024B digital oscilloscope was used to measure the frequency of the input drive voltage, while an Agilent 34461A digital multimeter was used to measure the output signal of the laser position detection system. An ac function generator was used to generate a swept frequency drive signal from $100 \mathrm{~Hz}$ to $3 \mathrm{kHz}$. The actuator was tested to have its first resonance at $\sim 2.61 \mathrm{kHz}$, as shown in Figure 17. This is in close agreement of the predicted resonant frequency of $2705 \mathrm{~Hz}$ found by COMSOL simulation. 


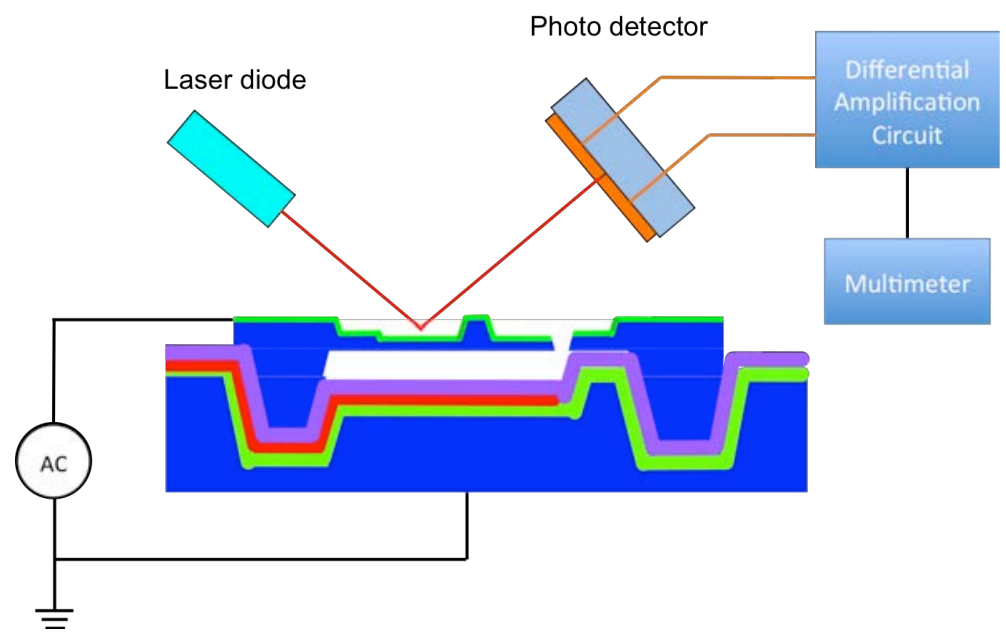

Figure 16. Set-up of the laser position measurement system.

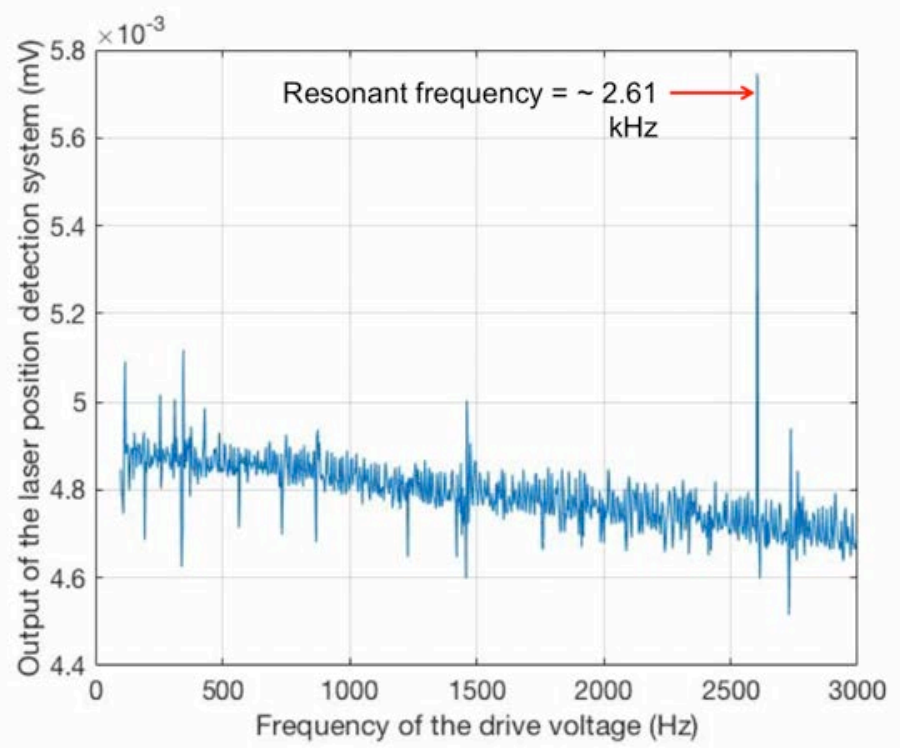

Figure 17. Resonant frequency test for the spiral actuator.

\section{CONCLUSION}

In this paper, we introduced a new electrostatic actuator design for double layer adaptive optics DM systems. The large stroke electrostatic actuator was designed with spiral support springs, attached to a small size and minimal mass central attachment. This new structure for electrostatic actuators, having Archimedean spiral springs which also serve as the electrode for electrostatic force coupling. This structure has advantages over conventional electrostatic actuators. It enables higher flexibility of the actuator within confined physical sizes, therefore reducing the required drive voltage for a specific stroke, without sacrificing the resonant frequency of the actuator as the conventional designs would. This is valuable especially to the applications where high spatial frequency of the MEMS actuator array is required, such as actuator array of a micro deformable mirror. Second, the spring constant of the actuator is adjustable by increasing or reducing the number of spiral arms, while the resonant frequency of the actuator is largely independent to the number of the spiral arms. Third, the electrostatic pulling force scales with the number of spiral arms. Since the spring constant similarly scales with the number of spiral arms as well, it can be adjusted without affecting the required driving voltage for a given stroke. Combining all three features together, the actuator can be designed with its stiffness, the resonant frequency, and required drive voltage for a certain stroke independent to each other. By enabling these three specifications (stiffness, resonant frequency, drive voltage) to be treated independently in the design process, it provides wide latitude in exploring suitable performing designs. In the case of double layer deformable mirror with continuous phase sheet, it means reduction in the design complexity to reach a specific performance and compliance between adjacent locations of the phase sheet. The spiral actuator design was fabricated from a silicon membrane using laser 
micromachining. Experimental testing showed good agreement with simulations for the actuator spring constant and resonant frequency.

\section{ACKNOWLEDGMENT}

This project was financially supported by National Research Council Canada (NRC), and the Natural Sciences and Engineering Research Council of Canada (NSERC).

\section{REFERENCES}

[1] D. J. Bell, T. J. Lu, N. A. Fleck and S. M. Spearing, "MEMS actuators and sensors: observations on their performance and selection for purpose," J. Micromech. Microeng. 15, S153-164, (2005).

[2] T. Wu, M. Akiyama, and K. Hane, "Continuous membrane deformable mirror for adaptive optics using bimorph spring," Transducers'11, Beijing, China, June 5-9, (2011).

[3] Fangrong Hu, Jun Yao, Chuankai Qiu, Hao Ren, "A MEMS micromirror driven by electrostatic force," Journal of Electrostatics, vol. 68, pp. 237-242, (2010).

[4] Jen-Liang Wang, Tyng-Yow Chen, ChingWei Liu, Chen-Wei Edward Chiu, and Guo-Dung John Su, "Polymer deformable mirror for optical auto focusing," ETRI Journal, Vol. 29, 6, 817-819, (2007).

[5] Po-Yu Lin, Hsin-Ta Hsieh, and Guo-Dung John Su, "Design and fabrication of a large-stroke MEMS deformable mirror for wavefront control," J. Opt. 13 (2011) 055404, doi:10.1088/2040-8978/13/5/055404

[6] Sarah J. Lukes, and David L. Dickensheets, "SU-8 2002 surface micromachined deformable membrane mirrors," Journal of Microelectromechanical Systems 22, 1, 94-106, (2013).

[7] Wenying Ma, Changwei Ma, and Weimin Wang, "Surface micromachined MEMS deformable mirror based on hexagonal parallel-plate electrostatic actuator,” J. Phys.: Conf. Ser. 986 (2018) 012021, doi:10.1088/1742-6596/986/1/012021.

[8] Thomas G. Bifano, Raji Krishnamoorthy Mali, John Kyle Dorton, Julie Perreault, Nelsimar Vandelli, Mark N. Horenstein, and David A. Castanon, "Continuous-membrane surface-micromachined silicon deformable mirror," Opt. Eng. 36, 1354-1360, (1997).

[9] Kilo-DM Specification Sheet, Boston Micromachines Corporation, 2012.

[10]Il Woong Jung, Yves-Alain Peter, Emily Carr, Jen-Shiang Wang, and Olav Solgaard, "Single-Crystal-Silicon Continuous Membrane Deformable Mirror Array for Adaptive Optics in Space-Based Telescopes," IEEE Journal of Selected Topics In Quantum Electronics 13, 2, 162-167, (2007)

[11] Narito Shibaike, "Design of micro-mechanisms focusing on configuration, materials and processes," Materials \& Design 16.2, 83-90, (1995).

[12] William C. Tang, Tu-Cuong H. Nguyen, and Roger T. Howe, "Laterally driven polysilicon resonant microstructures," Sensors and actuators 20.1-2, 25-32, (1989).

[13] Yen-Ru Huang, Jing-Ru Huang, Yi-Min Chen, Ying-Sheng Huang, Gerd Keiser, San-Liang Lee, Kuei-Yi Lee. "Design and fabrication of a carbon-nanotube-based capacitor patterned on an Archimedean spiral," Solid State Communications 151.14, 1022-1024, (2011).

[14] Jinghong Chen, Jun Zou, Chang Liu, José E. Schutt-Ainé, and Sung-Mo (Steve) Kang, "Design and modeling of a micromachined high-Q tunable capacitor with large tuning range and a vertical planar spiral inductor," IEEE Transactions on Electron Devices 50.3, 730-739, (2003).

[15] Tetsuo Kan, Akihiro Isozaki, Natsuki Kanda, Natsuki Nemoto, Kuniaki Konishi, Hidetoshi Takahashi, Makoto Kuwata-Gonokami, Kiyoshi Matsumoto, and Isao Shimoyama, "Enantiomeric switching of chiral metamaterial for terahertz polarization modulation employing vertically deformable MEMS spirals," Nature communications 6, (2015).

[16] Chenye Yang, Sanwei Liu, Xin Xie, and Carol Livermore, "Compact, planar, translational piezoelectric bimorph actuator with Archimedes' spiral actuating tethers," Journal of Micromechanics and Microengineering 26.12, (2016).

[17] T. Fukushige, Y. Yokoyama, S. Hata, K. Masu, and A. Shimokohbe, "Fabrication and evaluation of an on-chip micro-variable inductuor," Microelectronic Engineering 67-68, 582-587, (2003).

[18] Takashi Fukushige, Seiichi Hata, and Akira Shimokohbe, "A MEMS conical spring actuator array," Journal of Microelectromechanical Systems 14, 2, 243-253, (2005).

[19] Fangrong Hu, Weiming Wang, and Jun Yao, "An electrostatic MEMS spring actuator with large stroke and out-of-plane actuation,” J. Micromech. Microeng. 21, 115029, (2011).

[20]Comsol Multiphysics ® Modeling Software, https://www.comsol.com/, (2017).

[21] Byoungyoul Park, Elnaz Afsharipour, Dwayne Chrusch, Cyrus Shafai, David Andersen, and Greg Burley, "A Low Voltage and Large Stroke Lorentz Force Continuous Deformable Polymer Mirror for Wavefront Control," Sensors and Actuators A 280, 197-204, (2018).

[22] E. S. Hung and S. D. Senturia, "Extending the travel range of analog-tuned electrostatic actuators," J. Microelectromech. Syst. 8, 497-505, (1999).

[23] A-355ps Operation Manual University of Manitoba, Oxford Lasers Ltd (C) Copyright 2013. 